\title{
On generalized strongly modified $h$-convex functions
}

\author{
Taiyin Zhao ${ }^{1}$, Muhammad Shoaib Saleem², Waqas Nazeer ${ }^{3 *}$, Imran Bashir² and ljaz Hussain²
}

"Correspondence:

nazeer.waqas@ue.edu.pk

${ }^{3}$ Department of Mathematics,

Government College University,

Lahore, Pakistan

Full list of author information is

available at the end of the article

\begin{abstract}
We derive some properties and results for a new extended class of convex functions, generalized strongly modified $h$-convex functions. Moreover, we discuss Schur-type, Hermite-Hadamard-type, and Fejér-type inequalities for this class. The crucial fact is that this extended class has awesome properties similar to those of convex functions.

Keywords: $h$-convex function; Modified $h$-convex function; Schur-type inequality; Hermite-Hadamard inequality; Fejér-type inequality
\end{abstract}

\section{Introduction}

Nowadays, in science and modern analysis the convexity plays an important role in economics, statistics, management science, engineering, and optimization theory. For instance, Barani et al. [1] presented the Hermite-Hadamard inequality for functions with preinvex absolute values of derivatives. Characterizations of convexity via Hadamard's inequality has been studied in [2]. In 2003, Dragomir and Pearce [3] proposed some applications of Hermite-Hadamard inequalities. In 2015, Dragomir [4] presented inequalities of Hermite-Hadamard type for $h$-convex functions on linear spaces. Some other interesting results can be found in books $[5,6]$ and research papers $[7,8]$. In the recent years, generalizations and extensions were made rapidly for convex functions; for a recent generalization, see [9-11].

Convexity in the classical sense for a function $g: L=\left[a_{1}, a_{2}\right] \subset \mathbb{R} \rightarrow \mathbb{R}$ is defined as

$$
g\left(t a_{1}+(1-t) a_{2}\right) \leq \operatorname{tg}\left(a_{1}\right)+(1-t) g\left(a_{2}\right)
$$

where $a_{1}, a_{2} \in L$ and $t \in[0,1]$.

The work on the convexity is extended day by day by using some techniques; see [12-14]. The strongly extended convexity is widely used in optimization, economics, and nonlinear programming.

Convex functiosn satisfy several inequalities in which famous inequalities are of Schur type, Hermite-Hadamard-type, and Fejér-type inequalities. The Hermite-Hadamardtype inequality introduced by Jaques Hadamard for classical convex functions $g: L=$

(c) The Author(s) 2020. This article is licensed under a Creative Commons Attribution 4.0 International License, which permits use, sharing, adaptation, distribution and reproduction in any medium or format, as long as you give appropriate credit to the original author(s) and the source, provide a link to the Creative Commons licence, and indicate if changes were made. The images or other third party material in this article are included in the article's Creative Commons licence, unless indicated otherwise in a credit line to the material. If material is not included in the article's Creative Commons licence and your intended use is not permitted by statutory regulation or exceeds the permitted use, you will need to obtain permission directly from the copyright holder. To view a copy of this licence, visit http://creativecommons.org/licenses/by/4.0/. 


$$
\begin{aligned}
& {\left[a_{1}, a_{2}\right] \subset \mathbb{R} \rightarrow \mathbb{R} \text { as }} \\
& \qquad g\left(\frac{a_{1}+a_{2}}{2}\right) \leq \frac{1}{a_{2}-a_{1}} \int_{a_{1}}^{a_{2}} g(x) d x \leq \frac{g\left(a_{1}\right)+g\left(a_{2}\right)}{2} .
\end{aligned}
$$

For extended versions of this inequality, see [12] and [13]. For further reading, see [15-19].

Lipot Fejér presented an extended version of the Hermite-Hadamard inequality, known as the Fejér inequality or a weighted version of the Hermite-Hadamard inequality. If $f$ : $I \rightarrow \mathbb{R}$ is a convex function, then

$$
g\left(\frac{a_{1}+a_{2}}{2}\right) \int_{a_{1}}^{a_{2}} w(x) d x \leq \frac{1}{a_{2}-a_{1}} \int_{a_{1}}^{a_{2}} w(x) g(x) d x \leq \frac{g\left(a_{1}\right)+g\left(a_{2}\right)}{2} \int_{a_{1}}^{a_{2}} w(x) d x,
$$

where $a_{1} \leq a_{2}$, and $w: I \rightarrow \mathbb{R}$ is nonnegative, integrable, and symmetric about $\frac{a+b}{2}$. For further extended versions and development, see [20] and [8].

In this paper, we first present some preliminaries and basic results. In the next section, we investigate Schur-type, Hermite-Hadamard-type, and Fejér-type inequalities for the newly introduced class of functions.

\section{Preliminaries}

In this section, we investigate a new class of convexity by using a basic result. There is no loss of generality in the extended version of convexity. To get asymptotic results, it is necessary to put some restrictions: $L$ is an interval in $\mathbb{R}$, and $\eta: A \times A \rightarrow B \subseteq \mathbb{R}$ is a bifunction.

Definition 1 ( $h$-convex function [21]) Let $g, h: L \subset \mathbb{R} \rightarrow \mathbb{R}$ be nonnegative functions. Then $g$ is called an $h$-convex function if

$$
g\left(t a_{1}+(1-t) a_{2}\right) \leq h(t) g\left(a_{1}\right)+h(1-t) g\left(a_{2}\right)
$$

for all $a_{1}, a_{2} \in L$ and $t \in[0,1]$.

Definition 2 (Modified $h$-convex function [13]) Let $g, h: L \subset \mathbb{R} \rightarrow \mathbb{R}$ be nonnegative functions. Then $g$ is called a modified $h$-convex function if

$$
g\left(t a_{1}+(1-t) a_{2}\right) \leq h(t) g\left(a_{1}\right)+(1-h(t)) g\left(a_{2}\right)
$$

for all $a_{1}, a_{2} \in L$ and $t \in[0,1]$.

Definition 3 (Generalized modified $h$-convex function) Let functions $g, h: J \subset \mathbb{R} \rightarrow \mathbb{R}$ be nonnegative functions. Then $g: I \subset \mathbb{R} \rightarrow \mathbb{R}$ is called a generalized modified $h$-convex function if

$$
g\left(t a_{1}+(1-t) a_{2}\right) \leq g\left(a_{2}\right)+h(t) \eta\left(g\left(a_{1}\right), g\left(a_{2}\right)\right)
$$

for all $a_{1}, a_{2} \in I$ and $t \in[0,1]$. 
Definition 4 (Wright-convex function [20]) A function $g: L \subset \mathbb{R} \rightarrow \mathbb{R}$ is said to be Wright-convex if

$$
g\left((1-t) a_{1}+t a_{2}\right)+g\left(t a_{1}+(1-t) a_{2}\right) \leq g\left(a_{1}\right)+g\left(a_{2}\right)
$$

for all $a_{1}, a_{2} \in L$ and $t \in[0,1]$.

Definition 5 (Additivity) A function $\eta$ is said to be additive if $\eta\left(x_{1}, y_{1}\right)+\eta\left(x_{2}, y_{2}\right)=\eta\left(x_{1}+\right.$ $x_{2}, y_{1}+y_{2}$ ) for all $x_{1}, x_{2}, y_{1}, y_{2} \in \mathbb{R}$; see [22] for more detail.

Definition 6 (Nonnegative homogeneity) A function $\eta$ is said to be nonnegatively homogeneous if $\eta\left(\lambda a_{1}, \lambda a_{2}\right)=\lambda \eta\left(a_{1}, a_{2}\right)$ for all $a_{1}, a_{2} \in \mathbb{R}$ and $\lambda \geq 0$.

Definition 7 (Supermultiplicativity [23]) A function $g: L \subset \mathbb{R} \rightarrow \mathbb{R}_{+}$is said to be a supermultiplicative function if $g\left(a_{1} a_{2}\right) \geq g\left(a_{1}\right) g\left(a_{2}\right)$ for all $a_{1}, a_{2} \in L, t \in[0,1]$.

Definition 8 (Similar-order functions [24]) Functions $f$ and $g$ are said to be of similar order on $L \subseteq \mathbb{R}$ if $\langle f(x)-f(y), g(x)-g(y)\rangle \geq 0$ for all $x, y \in L$.

Now we are going to introduce a new extended definition of convexity.

Definition 9 (Generalized strongly modified $h$-convex function) Let $g, h: L \subset \mathbb{R} \rightarrow \mathbb{R}$ be nonnegative functions. Then $g$ is called a generalized strongly modified $h$-convex function if

$$
g\left(t a_{1}+(1-t) a_{2}\right) \leq g\left(a_{2}\right)+h(t) \eta\left(g\left(a_{1}\right), g\left(a_{2}\right)\right)-\mu t(1-t)\left(a_{1}-a_{2}\right)^{2}
$$

for all $a_{1}, a_{2} \in L$ and $t \in[0,1]$.

\section{Remark 1}

1. Inequality (3) reduces to inequality (1) if $\mu=0$ and $\eta(x, y)=x-y$.

2. Definition (9) becomes the definition of a classical convex function when $\mu=0$, $\eta(x, y)=x-y$, and $h(t)=t$.

3. Inequality(3) reduces to inequality (2) when $\mu=0$.

4. If $h(t)=t$, then definition (9) reduces to the definition of a strongly generalized convex function [12].

Example 1 A function $g: L=\left[a_{1}, a_{2}\right] \subset \mathbb{R} \rightarrow \mathbb{R}$ is defined by $g(x)=x^{2}, \eta\left(a_{1}, a_{2}\right)=2 a_{1}+a_{2}$, and $h(t) \geq t$, then $\mathrm{g}$ is a generalized strongly modified $h$-convex function.

\section{Main results}

This section contains some basic and straightforward results. The following proposition shows the linearity of the class of generalized strongly modified $h$-convex functions.

Proposition 1 Let $f$ and $g$ be generalized strongly modified h-convex functions where $\eta$ is additive and nonnegatively homogeneous. Then for all $a, b \in \mathbb{R}$, af + bg is also a generalized strongly modified h-convex function. 
Proposition 2 Let $h_{1}, h_{2}$ be nonnegative functions on $L$ such that $h_{2}(t) \leq h_{1}(t)$. If $g$ is a generalized strongly modified $h_{2}$-convex function, then $g$ is also a generalized strongly modified $h_{1}$-convex function.

Proof As $g$ is generalized strongly modified $h$-convex function, for all $a_{1}, a_{2} \in L$ and $t \in$ $[0,1]$, we have

$$
\begin{aligned}
g\left(t a_{1}+(1-t) a_{2}\right) & \leq g\left(a_{2}\right)+h_{2}(t) \eta\left(g\left(a_{1}\right), g\left(a_{2}\right)\right)-\mu t(1-t)\left(a_{1}-a_{2}\right)^{2} \\
& \leq g\left(a_{2}\right)+h_{1}(t) \eta\left(g\left(a_{1}\right), g\left(a_{2}\right)\right)-\mu t(1-t)\left(a_{1}-a_{2}\right)^{2} .
\end{aligned}
$$

This completes the proof.

Remark 2 If $g$ is a generalized strongly modified $h_{1}$-convex and $h_{1}(t) \leq h_{2}(t)$, then $g$ is a generalized strongly modified $h_{2}$-convex function.

Proposition 3 Let $f$ be a linear function such that $f(x)-f(y)=x-y$, and let $g$ be a generalized strongly modified h-convex function. Then $g$ of is also a generalized strongly modified h-convex function.

Proof As $f$ is a linear function such that $f(x)-f(y)=x-y$ and $g$ is a generalized strongly modified $h$-convex function, for all $a_{1}, a_{2} \in L$ and $t \in[0,1]$, we get

$$
\begin{aligned}
(g \circ f)\left(t a_{1}+(1-t) a_{2}\right)= & g\left(t f\left(a_{1}\right)+(1-t) f\left(a_{2}\right)\right. \\
\leq & (g \circ f)\left(a_{2}\right)+h(t) \eta\left((g \circ f)\left(a_{1}\right),(g \circ f)\left(a_{2}\right)\right) \\
& -\mu t(1-t)\left(f\left(a_{1}\right)-f\left(a_{2}\right)\right)^{2} \\
= & (g \circ f)\left(a_{2}\right)+h(t) \eta\left((g \circ f)\left(a_{1}\right),(g \circ f)\left(a_{2}\right)\right) \\
& -\mu t(1-t)\left(a_{1}-a_{2}\right)^{2} .
\end{aligned}
$$

This shows that $g \circ f$ is a generalized strongly modified $h$-convex function.

Proposition 4 Let functions $g_{j}: L \subset \mathbb{R} \rightarrow \mathbb{R}$ be generalized strongly modified h-convex functions, $\sum_{j=1}^{m} \lambda_{j}=1$, and let $\eta$ be additive non-negatively homogeneous function. Then their linear combination $f: \mathbb{R} \rightarrow \mathbb{R}$ is also a generalized strongly modified h-convex function.

Proof As $g_{j}: L \subset \mathbb{R} \rightarrow \mathbb{R}$ be generalized strongly modified $h$-convex functions, for $a_{1}, a_{2} \in$ $L$ and $t \in[0,1]$, let

$$
f(x)=\sum_{j=1}^{m} \lambda_{j} g_{j}(x) .
$$


Set $x=\left(t a_{1}+(1-t) a_{2}\right)$. Then

$$
\begin{aligned}
f\left(t a_{1}+(1-t) a_{2}\right)= & \sum_{j=1}^{m} \lambda_{j} g_{j}\left(t a_{1}+(1-t) a_{2}\right) \\
\leq & \sum_{j=1}^{m} \lambda_{j} g_{j}\left(a_{2}\right)+h(t) \sum_{j=1}^{m} \lambda_{j} \eta\left(g_{i}\left(a_{1}\right), g_{i}\left(a_{2}\right)\right) \\
& -\mu t(1-t)\left(a_{1}-a_{2}\right)^{2} \sum_{j=1}^{m} \lambda_{j} \\
= & f\left(a_{2}\right)+h(t) \eta\left(\sum_{j=1}^{m} \lambda_{j} g_{i}\left(a_{1}\right), \sum_{j=1}^{m} \lambda_{j} g_{i}\left(a_{2}\right)\right) \\
& -\mu t(1-t)\left(a_{1}-a_{2}\right)^{2} \\
= & f\left(a_{2}\right)+h(t) \eta\left(f\left(a_{1}\right), f\left(a_{2}\right)\right)-\mu t(1-t)\left(a_{1}-a_{2}\right)^{2} .
\end{aligned}
$$

This completes the proof.

Corollary 1 Every generalized strongly modified h-convex function is a generalized modified convex function.

Proof Let $g$ be a generalized modified $h$-convex function. Then

$$
\begin{aligned}
g\left(t a_{1}+(1-t) a_{2}\right) & \leq g\left(a_{2}\right)+h(t) \eta\left(g\left(a_{1}\right), g\left(a_{2}\right)\right)-\mu t(1-t)\left(a_{1}-a_{2}\right)^{2} \\
& \leq g\left(a_{2}\right)+h(t) \eta\left(g\left(a_{1}\right), g\left(a_{2}\right)\right)
\end{aligned}
$$

for all $a_{1}, a_{2} \in L \subset \mathbb{R}$.

Corollary 2 If $g$ is generalized strongly convex function and $t \leq h(t)$, then $g$ is a generalized strongly modified h-convex function.

Theorem 1 (Schur-type inequality) Let $g: L \rightarrow \mathbb{R}$ be a generalized strongly modified $h$ convex function, let $h$ be a supermultiplicative function, and let $\eta: N \times N \rightarrow M$ be a bifunction for appropriate $A, B \subseteq \mathbb{R}$. Then for $a_{1}, a_{2}, a_{3} \in L$ such that $a_{1}<a_{2}<a_{3}$ and $a_{3}-a_{1}, a_{3}-a_{2}, a_{2}-a_{1} \in L$, we have the inequality

$$
\begin{gathered}
h\left(a_{3}-a_{1}\right) g\left(a_{2}\right) \leq h\left(a_{3}-a_{1}\right) g\left(a_{3}\right)+h\left(a_{3}-a_{2}\right) \eta\left(g\left(a_{1}\right), g\left(a_{2}\right)\right) \\
-\mu\left(a_{3}-a_{2}\right)\left(a_{2}-a_{1}\right) h\left(a_{3}-a_{1}\right)
\end{gathered}
$$

if and only if $g$ is a generalized strongly modified h-convex function.

Proof Let $a_{1}, a_{2}, a_{3} \in L \subset \mathbb{R}$ be such that $\frac{\left(a_{3}-a_{2}\right)}{\left(a_{3}-a_{1}\right)} \in(0,1) \subseteq L, \frac{\left(a_{2}-a_{1}\right)}{\left(a_{3}-a_{1}\right)} \in(0,1) \subseteq L$, and $\frac{\left(a_{3}-a_{2}\right)}{\left(a_{3}-a_{1}\right)}+\frac{\left(a_{2}-a_{1}\right)}{\left(a_{3}-a_{1}\right)}=1$. Then

$$
h\left(a_{3}-a_{1}\right)=h\left(\frac{a_{3}-a_{1}}{a_{3}-a_{2}}\left(a_{3}-a_{2}\right)\right) \geq h\left(\frac{a_{3}-a_{1}}{a_{3}-a_{2}}\right) h\left(a_{3}-a_{2}\right)
$$

as $h$ is supermultiplicative. 
Suppose $h\left(a_{3}-a_{2}\right) \geq 0$. Then by the definition of $g$ we have

$$
g(t x+(1-t) y) \leq g(y)+h(t) \eta(g(x), g(y))-\mu t(1-t)(x-y)^{2} .
$$

Inserting $\frac{\left(a_{3}-a_{2}\right)}{\left(a_{3}-a_{1}\right)}=t, x=a_{1}$, and $y=a_{3}$ into inequality (5), we obtain

$$
\begin{aligned}
g\left(\frac{\left(a_{3}-a_{2}\right)}{\left(a_{3}-a_{1}\right)} a_{1}+\left(1-\frac{\left(a_{3}-a_{2}\right)}{\left(a_{3}-a_{1}\right)}\right) a_{3}\right) \leq & g\left(a_{3}\right)+h\left(\frac{\left(a_{3}-a_{2}\right)}{\left(a_{3}-a_{1}\right)}\right) \eta\left(g\left(a_{1}\right), g\left(a_{3}\right)\right) \\
& -\mu\left(a_{3}-a_{2}\right)\left(a_{2}-a_{1}\right) \\
\leq & g\left(a_{3}\right)+\frac{h\left(a_{3}-a_{2}\right)}{h\left(a_{3}-a_{1}\right)} \eta\left(g\left(a_{1}\right), g\left(a_{3}\right)\right) \\
& -\mu\left(a_{3}-a_{2}\right)\left(a_{2}-a_{1}\right), \\
g\left(a_{2}\right) h\left(a_{3}-a_{1}\right) \leq & h\left(a_{3}-a_{1}\right) g\left(a_{3}\right) \quad \\
& +h\left(a_{3}-a_{2}\right) \eta\left(g\left(a_{1}\right), g\left(a_{3}\right)\right) \\
& -\mu\left(a_{3}-a_{2}\right)\left(a_{2}-a_{1}\right) h\left(a_{3}-a_{1}\right) .
\end{aligned}
$$

Conversely, suppose inequality (4) holds and insert $a_{1}=x, a_{2}=t x+(1-t) y$, and $a_{3}=y$ into inequality (4). Then we get

$$
\begin{gathered}
h(y-x) g(t x+(1-t) y) \leq h(y-x) g(y)+h(y-x) h(t) \eta(g(x), g(y)) \\
-\mu h(y-x) t(y-x)(1-t)(y-x), \\
g(t x+(1-t) y) \leq g(y)+h(t) \eta(g(x), g(y))-\mu t(1-t)(x-y)^{2} .
\end{gathered}
$$

This completes the proof.

\section{Remark 3}

1. By taking $h(t)=t$ in (4) it is reduced to aSchur-type inequality for generalized strongly convex functions.

2. If $\mu=0$ and $\eta(x, y)=x-y$, then (4) is reduced to a Schur-type inequality for modified $h$-convex functions; see [13].

Further, we will discuss the Hermite-Hadamard-type inequality for generalized strongly modified $h$-convex functions.

Theorem 2 (Hermit-Hadamard-type inequality) Let function $g: L \rightarrow \mathbb{R}$ be a generalized strongly modified h-convex function on $\left[a_{1}, a_{2}\right]$ with $a_{1}<a_{2}$. Then

$$
\begin{aligned}
g\left(\frac{a_{1}+a_{2}}{2}\right)-h\left(\frac{1}{2}\right) M_{\eta}+\frac{\mu}{12}\left(a_{2}-a_{1}\right)^{2} & \leq \frac{1}{a_{2}-a_{1}} \int_{a_{1}}^{a_{2}} g((x) d x \\
& \leq g\left(a_{2}\right)+N_{\eta}-\frac{\mu}{6}\left(a_{2}-a_{1}\right)^{2} .
\end{aligned}
$$


Proof Choosing $w=t a_{1}+(1-t) a_{2}$ and $z=(1-t) a_{1}+t a_{2}$, we have

$$
\begin{aligned}
g\left(\frac{a_{1}+a_{2}}{2}\right) & =g\left(\frac{w+z}{2}\right) \\
& =g\left(\frac{t a_{1}+(1-t) a_{2}+(1-t) a_{1}+t a_{2}}{2}\right) .
\end{aligned}
$$

Now by the definition of $g$ we have

$$
\begin{aligned}
g\left(\frac{a_{1}+a_{2}}{2}\right) \leq & g\left((1-t) a_{1}+t a_{2}\right)+h\left(\frac{1}{2}\right) \eta\left(g\left(t a_{1}+(1-t) a_{2}\right), g\left((1-t) a_{1}+t a_{2}\right)\right) \\
& -\mu \frac{1}{2}\left(1-\frac{1}{2}\right)\left(a_{2}-a_{1}\right)^{2}(2 t-1)^{2} .
\end{aligned}
$$

Integrating with respect to $t$ on $[0,1]$, we get

$$
\begin{aligned}
g\left(\frac{a_{1}+a_{2}}{2}\right) \leq & \int_{0}^{1} g\left((1-t) a_{1}+t a_{2}\right) d t \\
& +h\left(\frac{1}{2}\right) \int_{0}^{1} \eta\left(g\left(t a_{1}+(1-t) a_{2}\right), g\left((1-t) a_{1}+t a_{2}\right)\right) d t \\
& -\frac{\mu}{4}\left(a_{2}-a_{1}\right)^{2} \int_{0}^{1}(2 t-1)^{2} d t .
\end{aligned}
$$

Putting $x=(1-t) a_{1}+t a_{2}$, we get

$$
\begin{aligned}
& g\left(\frac{a_{1}+a_{2}}{2}\right) \leq \frac{1}{a_{2}-a_{1}} \int_{a_{1}}^{a_{2}} g(x) d x+h\left(\frac{1}{2}\right) M_{\eta}-\frac{\mu}{12}\left(a_{2}-a_{1}\right)^{2}, \\
& g\left(\frac{a_{1}+a_{2}}{2}\right)-h\left(\frac{1}{2}\right) M_{\eta}+\frac{\mu}{12}\left(a_{2}-a_{1}\right)^{2} \leq \frac{1}{a_{2}-a_{1}} \int_{a_{1}}^{a_{2}} g((x) d x .
\end{aligned}
$$

In the right-hand side of inequality (8), we set $x=t a_{1}+(1-t) a_{2}$, and using the definition of $g$, we get

$$
\begin{aligned}
& \int_{a_{1}}^{a_{2}} g(x) d x \leq\left(a_{2}-a_{1}\right) g\left(a_{2}\right)+\left(a_{2}-a_{1}\right) \int_{0}^{1} h(t) \eta\left(g\left(a_{1}, g\left(a_{2}\right)\right) d t-\frac{\mu}{6}\left(a_{2}-a_{1}\right)^{2},\right. \\
& \frac{1}{\left(a_{2}-a_{1}\right)} \int_{a_{1}}^{a_{2}} g(x) d x \leq g\left(a_{2}\right)+N_{\eta}-\frac{\mu}{6}\left(a_{2}-a_{1}\right)^{2} .
\end{aligned}
$$

Now from inequalities (8) and (9) we get

$$
\begin{aligned}
g\left(\frac{a_{1}+a_{2}}{2}\right)-h\left(\frac{1}{2}\right) M_{\eta}+\frac{\mu}{12}\left(a_{2}-a_{1}\right)^{2} & \leq \frac{1}{a_{2}-a_{1}} \int_{a_{1}}^{a_{2}} g((x) d x \\
& \leq g\left(a_{2}\right)+N_{\eta}-\frac{\mu}{6}\left(a_{2}-a_{1}\right)^{2}
\end{aligned}
$$

This completes the proof. 


\section{Remark 4}

1. If we take $\mu=0$ and $\eta(x, y)=x-y$, then the Hermite-Hadamard-type inequality (10) is reduced to Hermite-Hadamard-type inequality for modified $h$-convex functions; for details, see [13].

2. If we put $h(t)=t$ in (10), then we get a Hermite-Hadamard-type inequality for generalized strongly convex functions; see [12].

3. If we take $\mu=0, \eta(x, y)=x-y$ and $h(t)=t$, then inequality (10) is reduced to a Hemite-Hadard-type inequality for classical convex functions.

Now we prove the following lemma by using technique of [25]. This lemma has the crucial fact that generalized strongly modified $h$-convex functions behave like classic convex functions.

Lemma 1 Let $g$ be a generalized modified h-convex function, and suppose that $\eta(x, y)=$ $-\eta(y, x)$. Then

$$
g\left(a_{1}+a_{2}-x\right) \leq g\left(a_{1}\right)+g\left(a_{2}\right)-g(x) \quad \forall x \in\left[a_{1}, a_{2}\right]
$$

where $x=t a_{1}+(1-t) a_{2}$ and $t \in[0,1]$.

Proof As $g$ is generalized modified $h$-convex function, for $x=t a_{1}+(1-t) a_{2}$, we get

$$
\begin{aligned}
g\left(a_{1}+a_{2}-x\right) & =g\left((1-t) a_{1}+t a_{2}\right) \\
& \leq g\left(a_{1}\right)+h(t) \eta\left(g\left(a_{2}\right), g\left(a_{1}\right)\right) \\
& =g\left(a_{1}\right)+g\left(a_{2}\right)-g\left(a_{2}\right)-h(t) \eta\left(g\left(a_{1}\right), g\left(a_{2}\right)\right. \\
& =g\left(a_{1}\right)+g\left(a_{2}\right)-\left[g\left(a_{2}\right)+h(t) \eta\left(g\left(a_{1}\right), g\left(a_{2}\right)\right]\right. \\
& \leq g\left(a_{1}\right)+g\left(a_{2}\right)-g(x) .
\end{aligned}
$$

This completes the proof.

Lemma 2 Let $g$ be $q$ the generalized strongly modified h-convex function, and suppose that $\eta(x, y)=-\eta(y, x)$. Then

$$
g\left(a_{1}+a_{2}-x\right) \leq g\left(a_{1}\right)+g\left(a_{2}\right)-g(x) \quad \forall x \in\left[a_{1}, a_{2}\right],
$$

where $x=t a_{1}+(1-t) a_{2}$ and $t \in[0,1]$.

Proof Let $g$ be a generalized strongly modified $h$-convex function. Then for $x=t a_{1}+(1-$ $t) a_{2}$, we get

$$
\begin{aligned}
g\left(a_{1}+a_{2}-x\right)= & g\left((1-t) a_{1}+t a_{2}\right) \\
\leq & g\left(a_{1}\right)+h(t) \eta\left(g\left(a_{2}\right), g\left(a_{1}\right)\right)-\mu t(1-t)\left(a_{1}-a_{2}\right)^{2} \\
\leq & g\left(a_{1}\right)+g\left(a_{2}\right)-g\left(a_{2}\right)-h(t) \eta\left(g\left(a_{1}\right), g\left(a_{2}\right)\right. \\
& -\mu t(1-t)\left(a_{1}-a_{2}\right)^{2}+2 \mu t(1-t)\left(a_{1}-a_{2}\right)^{2}
\end{aligned}
$$




$$
\begin{aligned}
& \leq g\left(a_{1}\right)+g\left(a_{2}\right)-\left[g\left(a_{2}\right)+h(t) \eta\left(g\left(a_{1}\right), g\left(a_{2}\right)-\mu t(1-t)\left(a_{1}-a_{2}\right)^{2}\right]\right. \\
& \leq g\left(a_{1}\right)+g\left(a_{2}\right)-g(x) .
\end{aligned}
$$

This completes the proof.

It is very interesting that when $g$ is a modified $h$-convex function [13], generalized modified $h$-convex, or generalized strongly modified $h$-convex function, then inequality (11) holds.

Theorem 3 (Fejér-type inequality) Let $g:\left[a_{1}, a_{2}\right] \rightarrow \mathbb{R}$ be a generalized strongly modified $h$-convex, and let $w:\left[a_{1}, a_{2}\right] \rightarrow \mathbb{R}$ be nonnegative, integrable, and symmetric with respect to $\frac{a_{1}+a_{2}}{2}$. Then

$$
\begin{aligned}
& g\left(\frac{a_{1}+a_{2}}{2}\right) \int_{a_{1}}^{a_{2}} w(x) d x+\frac{\mu}{4} \int_{a_{1}}^{a_{2}}\left(a_{1}+a_{2}-2 x\right) w(x) d x-N_{\eta}\left(a_{1}, a_{2}\right) \\
& \quad \leq \int_{a_{1}}^{a_{2}} g(x) w(x) d x \\
& \quad \leq \frac{g\left(a_{1}\right)+g\left(a_{2}\right)}{2} \int_{a_{1}}^{a_{2}} w(x) d x+T_{\eta}\left(a_{1}, a_{2}\right)-\mu \int_{a_{1}}^{a_{2}}\left(x-a_{2}\right)\left(a_{1}-x\right) w(x) d x,
\end{aligned}
$$

where

$$
\begin{aligned}
& N_{\eta}\left(a_{1}, a_{2}\right)=h\left(\frac{1}{2}\right) \int_{a_{1}}^{a_{2}} \eta\left(g\left(a_{1}+a_{2}-x\right), g(x)\right) w(x) d x, \\
& T_{\eta}\left(a_{1}, a_{2}\right)=\frac{\eta\left(g\left(a_{1}\right), g\left(a_{2}\right)\right)}{2} \int_{a_{1}}^{a_{2}} h\left(\frac{x-a_{2}}{a_{1}-a_{2}}\right) w(x) d x .
\end{aligned}
$$

Proof Let $g$ be a generalized strongly modified $h$-convex function. Then

$$
\begin{aligned}
& g\left(\frac{a_{1}+a_{2}}{2}\right) \int_{a_{1}}^{a_{2}} w(x) d x= \int_{a_{1}}^{a_{2}} g\left(\frac{a_{1}+a_{2}-x+x}{2}\right) w(x) d x \\
& \leq \int_{a_{1}}^{a_{2}} g(x) w(x) d x \\
&+h\left(\frac{1}{2}\right) \int_{a_{1}}^{a_{2}} \eta\left(g\left(a_{1}+a_{2}-x\right), g(x)\right) w(x) d x \\
&-\int_{a_{1}}^{a_{2}} \mu \frac{1}{2}\left(1-\frac{1}{2}\right)\left(2 x-a_{1}-a_{2}\right)^{2} w(x) d x, \\
& g\left(\frac{a_{1}+a_{2}}{2}\right) \int_{a_{1}}^{a_{2}} w(x) d x+ \frac{\mu}{4} \int_{a_{1}}^{a_{2}}\left(a_{1}+a_{2}-2 x\right)^{2} w(x) d x-N_{\eta}\left(a_{1}, a_{2}\right) \\
& \leq \int_{a_{1}}^{a_{2}} g(x) w(x) d x .
\end{aligned}
$$


In the right hand-side of inequality (13), put $x=t a_{1}+(1-t) a_{2}$. Then

$$
\begin{aligned}
\int_{a_{1}}^{a_{2}} g(x) w(x) d x=\left(a_{2}-a_{1}\right) & \int_{0}^{1} g\left(t a_{1}+(1-t) a_{2}\right) w\left(t a_{1}+(1-t) a_{2}\right) d t \\
\frac{1}{a_{2}-a_{1}} \int_{a_{1}}^{a_{2}} g(x) w(x) d x \leq & \int_{0}^{1} g\left(a_{2}\right) w\left(t a_{1}+(1-t) a_{2}\right) d t \\
& +\eta\left(g\left(a_{1}\right), g\left(a_{2}\right)\right) \int_{0}^{1} h(t) w\left(t a_{1}+(1-t) a_{2}\right) d t \\
& -\mu\left(a_{2}-a_{1}\right)^{2} \int_{0}^{1} t(1-t) w\left(t a_{1}+(1-t) a_{2}\right) d t .
\end{aligned}
$$

Similarly, if we put $x=t a_{2}+(1-t) a_{1}$ in the right-hand side of inequality (13), then we get the inequality

$$
\begin{aligned}
\frac{1}{a_{2}-a_{1}} \int_{a_{1}}^{a_{2}} g(x) w(x) d x \leq & \int_{0}^{1} g\left(a_{1}\right) w\left(t a_{2}+(1-t) a_{1}\right) d t \\
& +\eta\left(g\left(a_{2}\right), g\left(a_{1}\right)\right) \int_{0}^{1} h(t) w\left(t a_{2}+(1-t) a_{1}\right) d t \\
& -\mu\left(a_{2}-a_{1}\right)^{2} \int_{0}^{1} t(1-t) w\left(t a_{2}+(1-t) a_{1}\right) d t .
\end{aligned}
$$

Adding inequalities (14) and (15), where $w$ is symmetric, we get

$$
\begin{aligned}
& \frac{2}{a_{2}-a_{1}} \int_{a_{1}}^{a_{2}} g(x) w(x) d x \\
& \leq\left(g\left(a_{1}\right)+g\left(a_{2}\right)\right) \int_{0}^{1} w\left(t a_{1}+(1-t) a_{2}\right) d t \\
& \quad+\left[\eta\left(g\left(a_{1}\right), g\left(a_{2}\right)\right)+\eta\left(g\left(a_{2}\right), g\left(a_{1}\right)\right)\right] \int_{0}^{1} h(t) w\left(t a_{1}+(1-t) a_{2}\right) d t \\
& \quad-2 \mu\left(a_{2}-a_{1}\right)^{2} \int_{0}^{1} t(1-t) w\left(t a_{1}+(1-t) a_{2}\right) d t .
\end{aligned}
$$

Putting $x=t a_{1}+(1-t) a_{2}$ in the right-hand side of inequality (16), we have

$$
\begin{aligned}
\int_{a_{1}}^{a_{2}} g(x) w(x) d x \leq & \frac{\left(g\left(a_{1}\right)+g\left(a_{2}\right)\right)}{2} \int_{a_{1}}^{a_{2}} w(x) d x \\
& +\frac{\left[\eta\left(g\left(a_{1}\right), g\left(a_{2}\right)\right)+\eta\left(g\left(a_{2}\right), g\left(a_{1}\right)\right)\right]}{2} \int_{a_{1}}^{a_{2}} h\left(\frac{x-a_{2}}{a_{1}-a_{2}} w(x) d x\right. \\
& -\mu \int_{a_{1}}^{a_{2}}\left(x-a_{2}\right)\left(a_{1}-x\right) w(x) d x, \\
\int_{a_{1}}^{a_{2}} g(x) w(x) d x \leq & \frac{\left(g\left(a_{1}\right)+g\left(a_{2}\right)\right)}{2} \int_{a_{1}}^{a_{2}} w(x) d x+T_{\eta}\left(a_{1}, a_{2}\right) \\
& -\mu \int_{a_{1}}^{a_{2}}\left(x-a_{2}\right)\left(a_{1}-x\right) w(x) d x .
\end{aligned}
$$


Now from inequalities (13) and (17) we get Fejér-type inequality (12) for generalized strongly modified $h$-convex functions.

\section{Remark 5}

1. If $h(t)=t$, then inequality (12) reduced to Fejér type inequality for generalized strongly convex functions, see [12].

2. If we put $\mu=0$ and $\eta(x, y)=x-y$ then inequality (12) becomes a Fejér-type inequality for modified $h$-convex functions; see [13].

3. If we put $\mu=0, \eta(x, y)=x-y$, and $h(t)=t$, then inequality (12) is reduced to a Fejér-type inequality for classical convex functions.

\section{Funding}

Not applicable.

\section{Availability of data and materials}

Data are included within this paper.

\section{Competing interests}

Authors of this paper declare that they have no competing interests.

\section{Authors' contributions}

All authors have equal contribution. All authors read and approved the final manuscript.

\section{Author details}

'School of Information and Software Engineering, University of Electronic Science and Technology of China, Chengdu, China. ${ }^{2}$ Department of Mathematics, University of Okara, Okara, Pakistan. ${ }^{3}$ Department of Mathematics, Government College University, Lahore, Pakistan.

\section{Publisher's Note}

Springer Nature remains neutral with regard to jurisdictional claims in published maps and institutional affiliations.

Received: 20 July 2019 Accepted: 6 January 2020 Published online: 15 January 2020

\section{References}

1. Barani, A., Ghazanfari, A.G., Dragomir, S.S.: Hermite-Hadamard inequality for functions whose derivatives absolute values are preinvex. J. Inequal. Appl. 2012(1), 247 (2012)

2. Bessenyei, M., Páles, Z:: Characterizations of convexity via Hadamard's inequality. Math. Inequal. Appl. 9(1), 53-62 (2006)

3. Dragomir, S.S., Pearce, C.: Selected topics on Hermite-Hadamard inequalities and applications. Math. Prepr. Arch. 2003(3), 463-817 (2003)

4. Dragomir, S.S.: Inequalities of Hermite-Hadamard type for $h$-convex functions on linear spaces. Proyecciones 34(4), 323-341 (2015)

5. Hardy, G.H., Littlewood, J.E., Polya, G.: Inequalities. Cambridge University Press, Cambridge (1952) (1988)

6. Kuczma, M.: An Introduction to the Theory of Functional Equations and Inequalities: Cauchy's Equation and Jensen's Inequality. Springer, Berlin (2009)

7. Merentes, N., Nikodem, K.: Remarks on strongly convex functions. Aequ. Math. 80(1-2), 193-199 (2010)

8. Tseng, K.L., Hwang, S.R., Dragomir, S.S.: Fejér-type inequalities (I). J. Inequal. Appl. 2010 (1), 531976 (2010)

9. Niculescu, C., Persson, L.E.: Convex Functions and Their Applications. Springer, New York (2006)

10. Peajcariaac, J.E., Tong, Y.L.: Convex Functions, Partial Orderings, and Statistical Applications. Academic Press, San Diego (1992)

11. Zalinescu, C.: Convex Analysis in General Vector Spaces. World Scientific, Singapore (2002)

12. Awan, M.U.: On strongly generalized convex functions. Filomat 31(18), 5783-5790 (2017)

13. Noor, M., Noor, K., Awan, U.: Hermite-Hadamard type inequalities for modified $h$-convex functions. Transylv. J. Math. Mech. 2014, 6 (2014)

14. Noor, M.A., Noor, K.I., Iftikhar, S., Awan, M.U.: Strongly generalized harmonic convex functions and integral inequalities. J. Math. Anal. 7(3), 66-71 (2016)

15. Bessenyei, M., Páles, Z.: Hermite-Hadamard inequalities for generalized convex functions. Aequ. Math. 69(1), 32-40 (2005)

16. Mitrinovic, D.S., Vasic, P.M.: Analytic Inequalities (Vol. 1). Springer, Berlin (1970)

17. Mordukhovich, B.S., Nam, N.M.: An easy path to convex analysis and applications. Synth. Lect. Math. Stat. 6(2), 1-218 (2013)

18. Rockafellar, R.T.: Convex Analysis (Vol. 28). Princeton University press, Princeton (1970)

19. Zabandan, G., Bodaghi, A., Kiliçman, A.: The Hermite-Hadamard inequality for $r$-convex functions. Journal of Inequalities and Applications 2012(1), 215 (2012) 
20. Ciobotariu-Boer, V:: Hermite-Hadamard and Fejér inequalities for Wright-convex functions. Octogon Math. Mag. 17(1), 53-69 (2009)

21. Varosanec, S.: On h-convexity. J. Math. Anal. Appl. 326(1), 303-311 (2007)

22. Shaikh, A.A., labal, A., Mondal, C.K.: Some results on $\varphi$-convex functions and geodesic $\varphi$-convex functions. Differ. Geom. Dyn. Syst. 20, 159-170 (2018)

23. Finol, C.E., Wojtowicz, M.: Multiplicative properties of real functions with applications to classical functions. Aequ. Math. 59(1-2), 134-149 (2000)

24. Peajcariaac, J.E., Tong, Y.L.: Convex Functions, Partial Orderings, and Statistical Applications. Academic Press, San Diego (1992)

25. Sarikaya, M.Z., Set, E., Ozdemir, M.E.: On some new inequalities of Hadamard type involving $h$-convex functions. Acta Math. Univ. Comen. 79(2), 265-272 (2010)

Submit your manuscript to a SpringerOpen ${ }^{\circ}$ journal and benefit from:

- Convenient online submission

- Rigorous peer review

- Open access: articles freely available online

- High visibility within the field

- Retaining the copyright to your article

Submit your next manuscript at $\gg$ springeropen.com 